\title{
Kommentar
}

Schweiz. Zschr. GanzheitsMedizin 17, 196-197 (2005). @ Verlag für GanzheitsMedizin, Basel. www.ganzheitsmedizin.ch

\section{Silva Keberle}

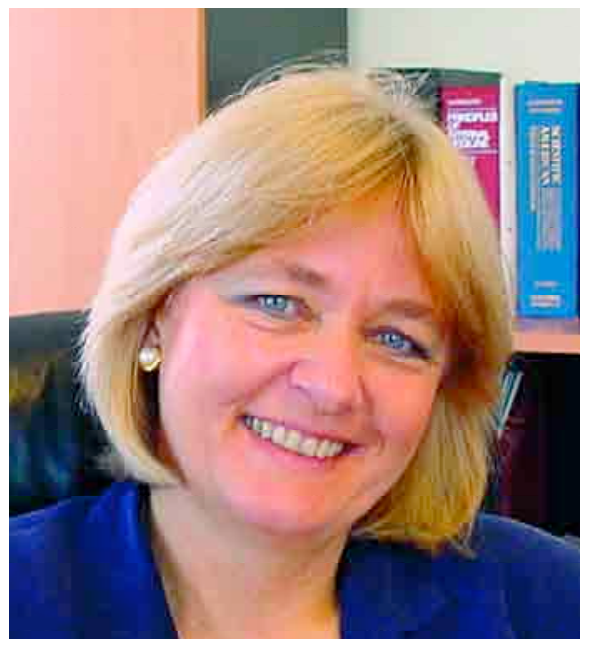

\section{Das ErfahrungsMedizinische Register (EMR)}

\author{
Ein wichtiger Beitrag zur Qualitätssicherung \\ in der Komplementärmedizin
}

A lle therapeutisch tätigen Personen beschäftigen sich mit der Gesundheit ihrer Patienten. Therapeutinnen und Therapeuten haben die schwierige Aufgabe, Beschwerden und Krankheiten zu diagnostizieren, die richtige Behandlung zu wählen und diese auch durchzuführen. Angesichts der bedeutenden Tatsache, dass die Gesundheit der behandelten Menschen auf dem Spiel steht, müssen an die Fähigkeiten und die Ausbildung von Therapeuten hohe Anforderungen gestellt werden. Dies gilt für Therapeuten der Komplementärmedizin ebenso wie für Therapeuten anderer medizinischer Richtungen.

Das ErfahrungsMedizinische Register (EMR), eine Abteilung der Eskamed AG in Basel, ist eine private, unabhängige, gesamtschweizerisch tätige Stelle, die ein Qualitätslabel im Bereich der Komplementärmedizin vergibt. Das EMR überprüft anhand von definierten Qualitätskriterien, den so genannten Registrierungsbedingungen, die Aus- und Fortbildung von komplementärmedizinisch tätigen Therapeuten. Therapeuten, welche die Registrierungsbedingungen des EMR erfüllen, werden vom EMR registriert.

Den meisten komplementärmedizinisch tätigen Ärzten und Therapeuten ist das EMR ein Begriff. Allerdings wissen viele nicht, welche Ziele das EMR hat, wie das EMR arbeitet und was das EMR-Label bedeutet. Im Folgenden werden deshalb die häufigsten Fragen rund ums EMR beantwortet.

\section{Was leistet das EMR?}

Der Markt der Komplementärmedizin ist sehr heterogen. Neben hoch qualifizierten Therapeuten, die teilweise eine mehrjährige Ausbildung oder gar ein Studium absolviert haben, bieten auch Therapeuten ihre Dienste an, die nur über eine minimale oder sogar gar keine Ausbildung verfügen. In der öffentlichen Wahrnehmung werden oft alle komplementärmedizinisch tätigen Therapeuten in einen Topf geworfen, unabhängig von ihrer Qualifikation. Durch die Vergabe eines Qualitätslabels leistet das EMR einen Beitrag zur Qualitätssicherung im Bereich der Komplementärmedizin. Denn zum Schutz von Patienten und Klienten ist es wichtig, dass Komplementärtherapeuten über eine gute Ausbildung verfügen, auf ihrem Gebiet praktische Erfahrungen gesammelt haben und sich auch nach Abschluss der Ausbildung regelmässig fortbilden. Ausbildung, Erfahrung und Fortbildung sind denn auch die wichtigsten Registrierungsbedingungen des EMR.

\section{Wie arbeitet das EMR?}

Das EMR überprüft anhand von Dokumenten, die jeder einzelne Therapeut einreicht, dass der Therapeut über eine abgeschlossene, methodenspezifische und schulmedizinische Ausbildung verfügt, dass er praktische Erfahrungen erworben hat und dass er sich kontinuierlich fortbildet. Das EMR-Team besteht aus einem Tages- 
und Abendteam. Das Tagesteam, insgesamt 16 Personen, ist in erster Linie für konzeptionelle und administrative Fragen zuständig. Es hält den Kontakt zu den Fachgremien des EMR und zu den Versicherern und überprüft komplexe Anmeldeunterlagen. Das Abendteam besteht aus 16 bis 20 Sachbearbeitern; sie kontrollieren die eingereichten Anmeldeunterlagen, geben die Daten in die Datenbank des EMR ein und beantworten an der Hotline Fragen der Therapeuten. Viele Mitglieder des EMR-Teams verfügen selbst über schul- oder komplementärmedizinische Ausbildungen.

\section{Wer nutzt das EMR-Label?}

Die Kosten von komplementärmedizinischen Leistungen werden heute mit Ausnahme weniger Methoden teilweise über die Zusatzversicherungen vergütet. Deshalb haben die Versicherer ein grosses Interesse an Systemen, mit denen die Qualität der Aus- und Fortbildung von Therapeuten sichergestellt werden kann. Das EMR ist ein solches Qualitätssicherungssystem, indem es ein Qualitätslabel zur Verfügung stellt. Therapeuten mit einer EMR-Registrierung werden in die EMR-Therapeutenliste aufgenommen. Eine grössere Zahl von Versicherern stellt bei der Vergütung von komplementärmedizinischen Leistungen im Rahmen der Zusatzversicherung auf die EMR-Therapeutenliste ab. Das bedeutet aber nicht, dass Therapeuten, die auf der EMR-Therapeutenliste stehen, automatisch mit Rückerstattungen ihrer Leistungen rechnen können. Die Versicherer nutzen zwar die EMRTherapeutenliste, sie sind aber völlig frei $\mathrm{zu}$ entscheiden, ob sie einzelne Therapeuten als Leistungserbringer anerkennen und in welchem Umfang sie deren Rechnungen rückerstatten wollen. Auf diese Entscheidungen der Versicherer hat das EMR keinen Einfluss.

\section{Was macht das EMR nicht?}

Das EMR ist ein unabhängiges Qualitätssicherungssystem. Das EMR führt selbst keine Ausbildungen, Fortbildungen oder Prüfungen durch. Das hat den Vorteil, dass das EMR allen Therapeuten offen steht, unabhängig davon, welche Schulen ein Therapeut absolviert hat oder welchen Berufsverbänden er angehört. Für alle Therapeuten gelten die gleichen Qualitätskriterien.

\section{Woher nimmt das EMR die Kompetenz?}

Das EMR arbeitet eng mit Sachverständigen der Versicherer, mit Vertretern von komplementärmedizinischen Schulen und Verbänden und mit weiteren Experten des Gesundheitswesen zusammen. Bei der Definition der Registrierungsbedingungen, der Erarbeitung von neuen Konzepten, der Beurteilung von komplexen oder problematischen Anmeldeunterlagen sowie Rekursen wird das EMR durch drei verschiedene beratende Gremien unterstützt. Es sind dies die «TaskForc», die sich aus Experten der Versicherer und erfahrenen Vertretern der Komplementärmedizin zusammensetzt, der so genannte «Runde Tisch» mit ebenfalls ausgewiesenen Therapeuten der Komplementärmedizin und der Fachbeirat unter dem Präsidium von Prof. Dr. med. Reinhard Saller, Dept. Innere Medizin / Naturheilkunde, UniversitätsSpital Zürich.

\section{Wer lässt sich beim EMR registrieren?}

Das EMR registriert Therapeuten, die eine oder mehrere von 143 Methoden ausüben, die momentan auf der Methodenliste des EMR stehen. Die Methodenliste entstand in Zusammenarbeit mit Vertretern von Versicherern und Experten der Komplementärmedizin; sie wird in regelmässigen Abständen überprüft und den neusten Erkenntnissen und Anforderungen angepasst. Die 143 Methoden auf der Methodenliste reichen von A wie Aderlass, Akumattherapie und Akupunktur bis W wie Wickel und WirbelsäulenBasis-Therapie.

\section{In welchem Rahmen lassen sich Ärzte beim EMR registrieren?}

Beim EMR werden vor allem nichtärztliche Therapeuten registriert. Die Versicherer verwenden das EMRLabel ausschliesslich im Bereich der Zusatzversicherungen, deshalb macht eine EMR-Registrierung für diejenigen Ärzte keinen Sinn, die mit einer der fünf für die Grundversicherung zugelassenen Komplementärmethoden arbeiten. Von den derzeit rund 13'500 registrierten Therapeuten sind knapp 200 Ärzte, also nur 1,5 Prozent. Beim EMR registrieren lassen sich zurzeit vor allem Ärzte aus dem Ausland, die in der Schweiz nie schulmedizinisch gearbeitet haben, oder Kollegen, die andere als die fünf für die Grundversicherung zugelassenen Methoden praktizieren.

Weitere Informationen zum EMR unter www.emr.ch

\section{Anschrift der Autorin:}

Dr. med. Silva Keberle Eskamed AG Postfach 158, CH-4011 Basel skeberle@eskamed.ch 